\title{
Recurrent Episodes of ST-Elevation Myocardial Infarction in a 19-Year-Old Male with Fontan Circulation
}

\author{
Alghammass MA ${ }^{1 *}$, Drakos SG ${ }^{1}$, Lal AK², Martinez \\ $\mathrm{HD}^{\mathbf{3}}$ and Kemeyou $\mathrm{L}^{1}$ \\ ${ }^{1}$ Division of Cardiovascular Medicine, Department of \\ Internal Medicine, University of Utah, USA \\ ${ }^{2}$ Division of Pediatric Cardiology, Department of \\ Pediatrics, University of Utah, USA \\ ${ }^{3}$ Division of Palliative Care, University of Utah, USA \\ *Corresponding author: Alghammass MA, \\ Department of Internal Medicine, Division of \\ Cardiovascular Medicine, University of Utah, $30 \mathrm{~N}$ \\ Medical Dr. Room 4a100, Salt Lake City, Utah 84132, \\ USA
}

Received: May 24, 2021; Accepted: J une 12, 2021;

Published: June 19, 2021

\section{Ablbreviations}

ECG: Electrocardiogram; LAD: Left Anterior Descending Artery; STEMI: ST-Segment Elevation Myocardial Infarction; DOAC: Direct Oral Anticoagulants; CVICU: Cardiovascular Intensive Care Unit

\section{Case Presentation}

19-year-old male with a history of single ventricle physiology (S,L,L) including L-transposition of the great arteries, pulmonary valve atresia, left atrioventricular valve atresia, large unrestrictive VSD, unrestrictive secundum ASD, and left pulmonary artery stenosis status post staged corrective heart surgery with eventually extracardiac Fontan at the age of two who presented to our emergency department complaining of abdominal pain for five days, associated with nausea and vomiting. Prior to his presentation to our facility, he was recently discharged from a local hospital after evaluation of chest pain with left sided weakness; he was found to have severe systolic and diastolic dysfunction, and age indeterminate thromboembolic stroke. His cardiac catheterization during this admission did not show any coronary vasculature abnormalities. He was discharged on aspirin, furosemide and rivaroxaban due to patient's refusal to be treated with warfarin. His medications history also includes daily prophylactic aspirin since his Fontan procedure, but he reported not taking it.

On exam he was vitally stable with left upper quadrant tenderness. Labs notable for normal complete blood count and kidney function. B-type natriuretic peptide resulted $1235 \mathrm{pg} / \mathrm{mL}$ (normal range: $\leq 100 \mathrm{pg} / \mathrm{mL}$ ). Chest radiograph showed evidence of pulmonary edema and CT abdomen/pelvis revealed acute splenic infarct and left renal infarct. He was admitted to the Cardiovascular Intensive Care Unit (CVICU) for management of acute exacerbation of heart failure, and acute infarcts involving the spleen and left renal arteries. He was started on infusions of milrinone and furosemide; as well as heparin infusion for management of heart failure and thromboemboli respectively. Our adult congenital heart disease and hematology teams were also involved in his inpatient care.

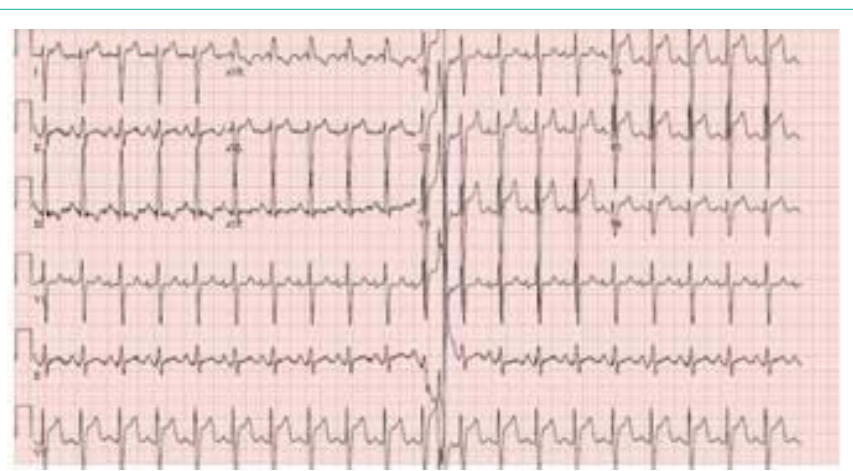

Figure 1: Electrocardiogram.

Electrocardiogram depicting anterolateral ST-Segment Elevation Myocardial Infarction (STEMI).

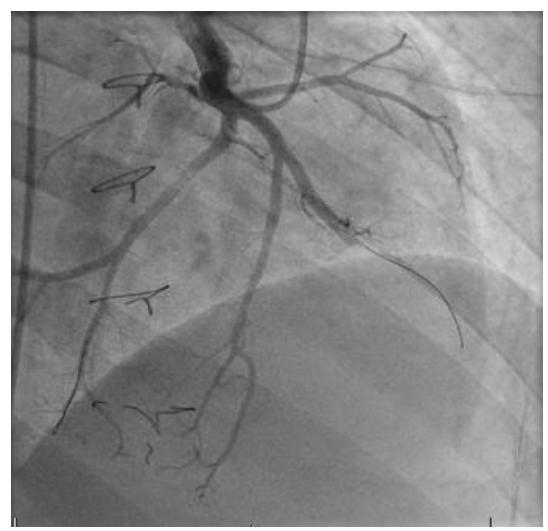

Figure 2: Coronary angiogram.

Angiography of the morphologic left coronary artery demonstrating a cutoff in the mid Left Anterior Descending artery (LAD).

Shortly following admission, he reported acute severe nonradiating substernal pressure-like chest pain. Clinical examination was notable for tachycardia. Electrocardiogram (ECG) showed
Austin J Clin Cardiolog - Volume 7 Issue 2 - 2021 ISSN : 2381-9111 | www.austinpublishing group.com Alghammass et al. @ All rights are reserved
Citation: Alghammass MA, Drakos SG, Lal AK, Martinez HD and Kemeyou L. Recurrent Episodes of ST-Elevation Myocardial Infarction in a 19-Year-Old Male with Fontan Circulation. Austin J Clin Cardiolog. 2021; 7(2): 1078. 


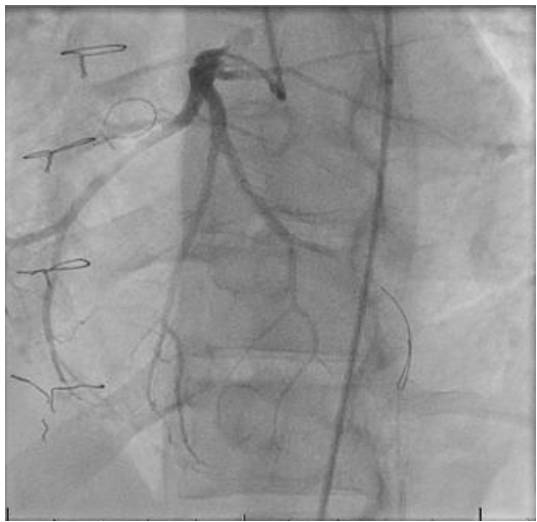

Figure 3: Coronary angiogram.

Angiography post thrombectomy with blood flow reestablished to the Left Anterior Descending Artery (LAD); the terminal vessel remained occluded.

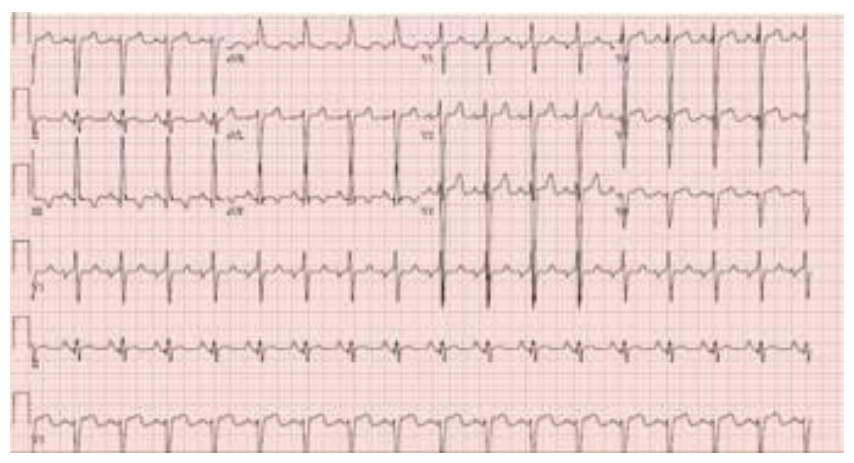

Figure 4: Electrocardiogram.

Electrocardiogram showing resolution of ST-segment elevations in the anterolateral leads following thrombectomy.

ST elevation in the anterolateral leads (Figure 1). Patient was immediately taken to the cardiac catheterization laboratory where coronary angiogram showed situs inversus, and the morphologic left coronary artery demonstrated a thrombotic occlusion in the mid Left Anterior Descending Artery (LAD) (Figure 2). A thrombectomy was performed and coronary flow was re-established; however, this intervention did not restore distal flow. Stenting was not performed given the embolic nature of the lesion. The final angiography shot showed TIMI3 flow in all vessels with persistent limited flow and occlusion in the distal LAD (Figure 3). The patient was transferred back to the CVICU and continued on antiplatelet therapy, with resolution of symptoms and ECG changes (Figure 4). Troponin peaked at $16.5 \mathrm{ng} / \mathrm{mL}$ (normal range: $\leq 0.03 \mathrm{ng} / \mathrm{mL}$ ).

Following clinical stabilization, a cardiac MRI performed with contrast showed globular, hypointense filling defect at the apex of the systemic ventricle $(1.2 \mathrm{~cm} \times 0.8 \mathrm{~cm})$, consistent with thrombus (Figure 5). Additional work up was unrevealing (including causes for a hypercoagulable state). He was eventually transferred out of the CVICU to the telemetry cardiology ward in a stable condition. Four days following the initial ST-Segment Elevation Myocardial Infarction (STEMI) episode, he again developed acute chest pain and ECG showed lateral STEMI. However, a repeat coronary angiogram demonstrated no change and subsequently the ECG normalized. Given the patient's refusal to be treated with warfarin, he was

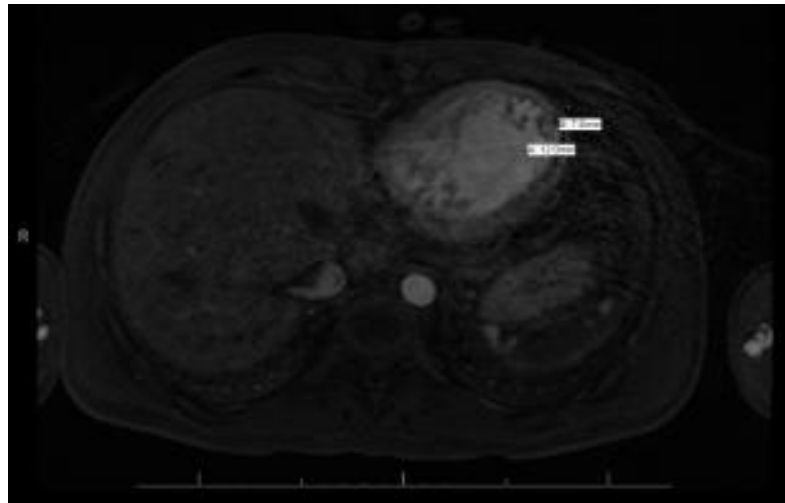

Figure 5: Cardiac Magnetic Resonance Imaging (MRI)

Cardiac MRI demonstrating globular, hypointense structure at the apex of the systemic ventricle $(1.2 \mathrm{~cm} \times 0.8 \mathrm{~cm})$ consistent with thrombus.

eventually transitioned to Direct Oral Anticoagulant (DOAC) and discharged home, as he remained chest pain free and stable.

\section{Discussion}

Fontan procedure is the final stage of surgical palliative approach to manage patients with single ventricle physiology, it is usually performed at 18 months to 4 years of age [1]. Following this procedure, multiple complications have been reported including but not limited to hepatic dysfunction, myocardial dysfunction, atrial rhythm disturbances, worsening cyanosis and thromboembolic events [2]. Thromboembolic events in particular impose high risk of morbidity and mortality with an incidence ranging from 3-20 \% [3]. The incidence of intracardiac thrombus remains unknown given the presence of asymptomatic cases, however it is reported as high as $33 \%$ [4]. There are only few reported cases of myocardial infarction as a result of thromboembolism in patients with Fontan circulation [5].

The efficacy of primary prophylaxis of thromboembolic events in Fontan circulation patients remains a debatable issue. Lower death rates have been reported in patients with Fontan circulation who are anticoagulated or in whom antiplatelet therapy is used [6]. Studies showed no superiority when using anticoagulation with warfarin compared to antiplatelet, therefore current guidelines recommend using aspirin for all patients with history of Fontan procedure [1]. According to the 2018 AHA/ACC guidelines for the management of adults with congenital heart disease, current indications for anticoagulation with warfarin in patients with Fontan circulation include cases with atrial arrhythmias, residual atrial septal defects/ fenestrations or thromboembolic event [6].

The guidelines however do not specifically recommend the use of DOAC at present time given the scarcity of evidence in this area. Despite that, the published literature supports the safety and non-inferiority of DOAC when compared to warfarin in Fontan circulation patients [7-11].

Fortunately, the occurrence of STEMI following thrombus formation is extremely rare, however among reported cases, primary angiography with balloon dilation and thrombectomy is the main approach for treatment [5]. Our patient was taken to the catheterization laboratory following each STEMI episode and treated 
with thrombectomy following the first episode. On both occasions he was treated with aspirin, heparin and tirofiban infusion for 18 hours. These interventions resulted in resolution of symptoms. He was finally discharged home on Aspirin and DOAC.

\section{Conclusion}

In conclusion, further research is needed to investigate the role of DOAC in treatment and prevention of thromboembolic events in patients with Fontan circulation. Our case specifically drew attention to patient's history of non-adherence to therapy as it could play major role in the decision-making regarding anticoagulation.

\section{References}

1. Ohye RG, Schranz D, D'Udekem Y. Current Therapy for Hypoplastic Left Heart Syndrome and Related Single Ventricle Lesions. Circulation. 2016; 134: 1265-1279.

2. Freedom RM, Yoo S-J. Complications of the Fontan Procedure. In: Freedom RM, Yoo S-J, Mikailian M, Williams W, editors. Natural and Modified History of Congenital Heart Disease. Elmsford, New York, USA: Blackwell Publishing. 2003: 460-470

3. Firdouse M, Agarwal A, Chan AK, Mondal T. Thrombosis and thromboembolic complications in Fontan patients: a literature review. Clin Appl Thromb Hemost. 2014; 20: 484-492

4. Balling G, Vogt $M$, Kaemmerer $\mathrm{H}$, Eicken A, Meisner $\mathrm{H}$, Hess $\mathrm{H}$. Intracardiac Thrombus Formation after the Fontan Operation. J Thorac Cardiovasc Surg. 2000; 119: 745-751.
5. Hastings RS, McElhinney DB, Saric M, Ngai C, Skolnick AH. Embolic myocardial infarction in a patient with a Fontan circulation. World J Pediatr Congenit Heart Surg. 2014; 5: 631-634.

6. Stout KK, Daniels CJ, Aboulhosn JA, Bozkurt B, Broberg CS, Colman JM, et al. $2018 \mathrm{AHA} / \mathrm{ACC}$ guideline for the management of adults with congenital heart disease: a report of the American College of Cardiology/American Heart Association Task Force on Clinical Practice Guidelines. Circulation. 2019; 139: e698-e800.

7. Kawamatsu N, Ishizu T, Machino-Ohtsuka T, Masuda K, Horigome $\mathrm{H}$, Takechi $F$, et al. Direct oral anticoagulant use and outcomes in adult patients with Fontan circulation: A multicenter retrospective cohort study. Int J Cardiol. 2020.

8. Kazerouninia A, Georgekutty J, Ermis P, Parekh D, Franklin W, Lam W. Novel oral anticoagulants, warfarin and aspirin in adult fontan patients: a single center experience. J Am Coll Cardiol. 2018; 71: A537.

9. Pujol C, Müssigmann M, Schiele S, Nagdyman N, Niesert AC, Kaemmerer $\mathrm{H}$, et al. Direct oral anticoagulants in adults with congenital heart disease - a single centre study. Int J Cardiol. 2020; 300: 127-131.

10. Yang H, Veldtman GR, Bouma BJ, Budts W, Niwa K, Meijboom F, et al. Nonvitamin $\mathrm{K}$ antagonist oral anticoagulants in adults with a Fontan circulation: are they safe. Open Heart. 2019; 6: e000985.

11. Georgekutty J, Kazerouninia A, Wang Y, Ermis PR, Parekh DR, Franklin WJ, et al. Novel oral anticoagulant use in adult Fontan patients: A single center experience. Congenit Heart Dis. 2018; 13: 541-547. 